\title{
Primary Biliary Cirrhosis: Family Stories
}

\author{
Daniel Smyk, ${ }^{1}$ Evangelos Cholongitas, ${ }^{2}$ Stephen Kriese, ${ }^{1}$ Eirini I. Rigopoulou, ${ }^{3}$ \\ and Dimitrios P. Bogdanos ${ }^{1}$ \\ ${ }^{1}$ Institute of Liver Studies, King's College London School of Medicine at King's College Hospital, Denmark Hill Campus, \\ London SE5 9RS, UK \\ ${ }^{2} 4$ th Department of Internal Medicine, Medical School of Aristotle University, Hippocration General Hospital of Thessaloniki, \\ 49 Konstantinopoleos Street, Thessaloniki 54642, Greece \\ ${ }^{3}$ Department of Medicine and Research Laboratory of Internal Medicine, University of Thessaly Medical School, Thessaly, \\ Mezourlo, Larissa 41222, Greece
}

Correspondence should be addressed to Dimitrios P. Bogdanos, dimitrios.bogdanos@kcl.ac.uk

Received 10 December 2010; Revised 9 February 2011; Accepted 7 March 2011

Academic Editor: I. R. Mackay

Copyright () 2011 Daniel Smyk et al. This is an open access article distributed under the Creative Commons Attribution License, which permits unrestricted use, distribution, and reproduction in any medium, provided the original work is properly cited.

\begin{abstract}
Primary biliary cirrhosis (PBC) is a chronic immune-mediated cholestatic liver disease of unknown aetiology which affects mostly women in middle age. Familial PBC is when PBC affects more than one member of the same family, and data suggest that firstdegree relatives of $\mathrm{PBC}$ patients have an increased risk of developing the disease. Most often, these familial clusters involve motherdaughter pairs, which is consistent with the female preponderance of the disease. These clusters provide evidence towards a genetic basis underlying PBC. However, clusters of nonrelated individuals have also been reported, giving strength to an environmental component. Twin studies have demonstrated a high concordance for PBC in monozygotic twins and a low concordance among dizygotic twins. In conclusion, studies of PBC in families clearly demonstrate that genetic, epigenetic, and environmental factors play a role in the development of the disease.
\end{abstract}

\section{Introduction}

Primary biliary cirrhosis $(\mathrm{PBC})$ is a chronic cholestatic liver disease characterised by an immune-mediated inflammatory destruction of the small intrahepatic bile ducts, progressing to fibrosis, cirrhosis, and subsequent liver failure [1-3]. The disease affects mainly middle-aged women and is practically absent in paediatric age [1-3]. More than $90 \%$ of the patients are women. The prevalence and incidence rates of the disease vary between different geographical areas. Studies from the United Kingdom suggested not only that $\mathrm{PBC}$ is the most frequent autoimmune liver disease (with the other two being autoimmune hepatitis and primary sclerosing cholangitis) but also that the disease affects up to 1 in 800 women over 40 years of age [4]. Increasing trends of the incidence and prevalence of the disease have been reported in UK, USA, and Australia [4-6]. It remains elusive whether this increase is true or just represents earlier recognition of the disease due to greater awareness and improved diagnostic tools [1-3]. PBC affects all ethnic groups but it appears to be more prevalent in women of Caucasian origin. There is little information about ethnic differences in PBC. A recent comprehensive analysis has reported differences of the clinical characteristics between Caucasian, African American, and Hispanic patients with PBC in the USA [7]. A significant number of racial minority patients were screened for the purposes of this study. Peters et al. [7] reported that the establishment of cirrhosis at clinical presentation is seen more frequently in non-Caucasian compared to Caucasian PBC patients. This difference could not be explained by demographic or serological features alone. It remains unclear whether increased awareness of $\mathrm{PBC}$ as a cause of chronic progressive cholestatic disease among Caucasian compared to nonCaucasian cases could explain the observed differences [7]. Of note, Hispanic and African American patients were of similar age with the Caucasian ones. Migration studies seem to indicate that migrants change their risk of $\mathrm{PBC}$ by adapting to that of the population into which they move. 
This has lead to the appreciation that environmental factors play an important role in the development of the disease $[5,8,9]$.

The reason for the female predominance of $\mathrm{PBC}$ remains unclear. Other autoimmune diseases with such strong female preponderance are Sjögren's syndrome and autoimmune thyroiditis, two autoimmune diseases which frequently cooccur with $\mathrm{PBC}[1-3]$. There is no convincing reason which can explain why these among other autoimmune diseases are most frequently seen in cases with $\mathrm{PBC}$.

Patients with PBC at diagnosis can be either asymptomatic with normal biochemistry tests or asymptomatic with abnormal biochemical blood tests, symptomatic, or finally can have very advanced liver disease [1-3]. Presentation tends to be early and the diagnosis of $\mathrm{PBC}$ most often is made when the patient is still asymptomatic with an abnormal cholestatic liver biochemistry and an immunological profile compatible with the disease which is discovered at a routine check [1-3]. The most frequent presenting symptoms in patients with $\mathrm{PBC}$ are fatigue and pruritus, and patients may be referred initially to dermatologists, endocrinologists, or neurologists [1-3]. The cause of pruritus and fatigue remains unknown and does not always correlate with the severity of the disease. Pruritus appears to be worse amongst Hispanic and African Americans but the reasons for that remain largely unknown [7]. Osteoporosis may be the initial observation with no other symptoms or signs of liver disease. In most cases, the disease advances in a slow pace but symptoms of portal hypertension and hepatic decompensation (jaundice, ascites, or variceal bleeding) can develop several years after the original diagnosis [1-3].

The diagnosis of $\mathrm{PBC}$ is based on three widely accepted criteria: biochemical signs of cholestasis, presence of diseasespecific autoantibodies, and diagnostic histological features [1-3]. Histological features typical for PBC include destruction of biliary epithelial cells (BEC) and loss of small bile ducts with portal inflammatory cell infiltration or less frequently granulomas [1-3]. Early serological markers of cholestasis include increased levels of alkaline phosphatase and $\gamma$ GT. High levels of IgM may be present but the most prominent immunological feature of the disease is the presence of diagnostically-relevant, high-titre antibodies against mitochondrial (AMA) and nuclear (ANA) antigens [1-3, 29-34]. While AMA do not appear to have clinical significance, disease-specific ANA can identify a subgroup of PBC cases with more advanced disease [29, 30, 34-45]. A considerable proportion of patients may present with no increase of IgM or may turn negative for autoantibodies, with the latter finding largely depending on the sensitivity of the tests used for their routine autoantibody detection $[32,33,46,47]$.

Disease-specific AMA are directed against components of the 2-oxo-acid dehydrogenase complexes (formerly known as M2 antigen) and in particular the E2 subunits of the pyruvate dehydrogenase complex (PDC), branchedchain 2-oxoacid dehydrogenase complex (BCOADC), and 2oxoglutarate dehydrogenase complex (OGDC) $[1,29,30,32$, 34]. More than $90 \%$ of AMA positive cases with $\mathrm{PBC}$ have detectable anti-PDC-E2 antibodies [1, 29]. All PBC cases reactive with PDC-E2 cross-react with the PDC E3 binding protein (E3BP) $[1,29]$. The E1 $\alpha$ and $\mathrm{E} 1 \beta$ subunits of PDC are subdominant targets $[1,29,30,32,34]$. AMA can be of the IgG, IgA, and IgM isotypes. High titre AMA-M2 of class IgG are found in up to $95 \%$ of patients but in less than $2 \%$ of pathological and healthy controls [1,29]. Nevertheless, various studies reported that the presence of AMA is much higher than the prevalence of $\mathrm{PBC}$ in the general population, indicating that AMA may precede the symptomatic onset of the disease [48]. Studies investigating the natural history of PBC have demonstrated that AMA-positive, asymptomatic patients often have histological features diagnostic of, or compatible with PBC [48-51]. Taken together, these findings indicate that AMA is a very powerful predictor of future development of PBC. As such, the question has been raised as to whether potential screening programmes for AMA should be adopted, especially for female relatives of PBC patients $[34,52]$. To date, no general consensus has been met.

As AMA are not the only disease-specific autoantibodies, there is an ongoing discussion as to whether diagnostic testing needs to incorporate assays for PBC-specific ANA [34]. Two PBC-specific ANA IFL patterns have been identified: autoantibodies giving the "multiple nuclear dots" pattern mainly target the nuclear body sp100 and PML proteins, and those giving the "nuclear membrane" (rim) recognise gp210, nup62 and other nuclear membrane proteins $[30,31,34$, 53]. Both ANA types can be found in approximately $30 \%$ of $\mathrm{PBC}$ patients and show a significant disease specificity $[30,31,53]$. These disease-specific patterns can be present in AMA-positive and AMA-negative asymptomatic individuals and also in family members of PBC patients. When these antibodies are present at diagnosis, they seem to be able to identify individuals who will develop advanced disease faster than those seronegative for these autoantibodies [54]. Antibodies against centromere are mainly present in PBC cases with concurrent CREST syndrome and their presence is associated with a more advanced disease $[40,55]$.

The mechanisms responsible for the induction of PBCspecific AMA and ANA responses are poorly understood [29, 56-61]. In vitro studies implicate antigen-specific B-, CD4, CD8 T-lymphocyte responses in the induction and/or maintenance of autoaggressive pathology $[51,57,58,62-$ 65]. Animal models resembling PBC suggest that both arms of innate and adaptive immunity contribute to the disease development $[57,66,67]$.

As for other autoimmune diseases, genetic, epigenetic, environmental, and infectious factors have been considered important for the development of the disease or its progression from early stages to more advanced, lifethreatening biliary epithelial cell destruction $[23-25,57,58$, 65-79]. Familial studies have provided evidence towards both genetic and environmental causes [26, 27, 68]. Recent genomic studies have provided data which correlates with the increased incidence of $\mathrm{PBC}$ in families, as well as the increased incidence of autoimmune disease in both $\mathrm{PBC}$ patients and their relatives [23-25]. In order to differentiate between environment and genes (or a combination of the two), twin studies have been considered essential as monozygotic twins would share the same genetic material 


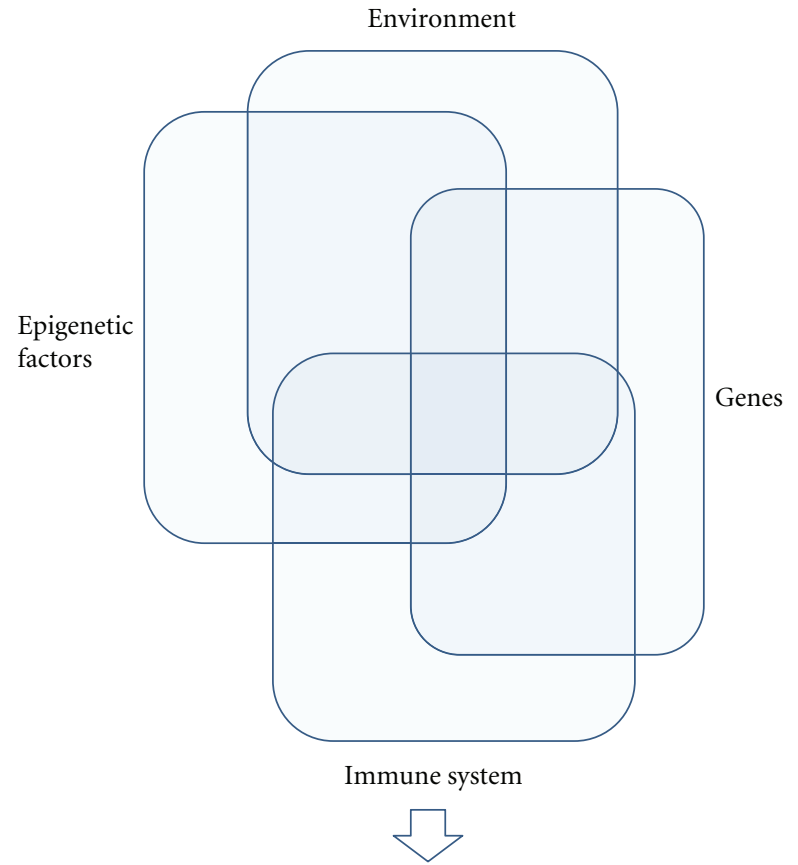

Biliary epithelial cell destruction-development of PBC

FIGURE 1: Understanding the pathogenesis of the disease also stems from studies of familial primary biliary cirrhosis (PBC). Clues as to the pathogenesis of the disease are provided by population studies involving a large number of families of affected individuals and case reports (see also Table 1). These studies support the notion that the pathogenesis of the disease is complex, and that genes, epigenetic factors, and environment are mostly likely involved in concert rather than in isolation. The relative contribution of each of those factors is largely unknown and may vary among individuals and clusters. Their participation may be important for the induction of the immunopathological processes leading to $\mathrm{PBC}$ development, but may also be important for the progression of the disease towards a favourable slow pace or an unwanted progressive deterioration.

and therefore have a high disease concordance. Likewise, dizygotic twins would share environmental factors, but differ genetically (Figure 1).

\section{Overview of Primary Biliary Cirrhosis in Families}

Like many autoimmune diseases, PBC infers an increased risk of developing the disease in relatives of an affected individual. Some of the major findings of the familial studies in PBC are summarised in Tables 1 and 2. Bach and Schaffner [10] at Mount Sinai Hospital in New York conducted a retrospective review to determine the prevalence of $\mathrm{PBC}$ within family members of PBC patients. Out of a total of 405 patients with $\mathrm{PBC}, 26$ also had at least one first-degree relative (FDR) who also had $\mathrm{PBC}$ or liver disease that was unspecified but suggestive of PBC [10]. The prevalence of $\mathrm{PBC}$ was calculated to be $4.3 \%$ in families with at least one relative with $\mathrm{PBC}$, or $14.9 \%$ when taking into account those with undiagnosed liver disease with some features
TABLe 1: Some clues for the aetiopathogenesis of primary biliary cirrhosis (PBC) are provided by family studies.

References

Genes

(a) High incidence of mother-daughter pairs, and sister-sister pairs

(b) Increased incidence in PBC rates in families of PBC patients

(c) Similar AMA reactivity profiles shared among affected relatives

(d) HLA DR8, the allele associated with susceptibility to $\mathrm{PBC}$ is found in familial cases

(e) Increased incidence of autoimmunity in PBC patients and their families

(f) Identification of non-HLA genes with increased incidence among PBC patients (e.g., STAT4, CTLA4)

(g) Increased concordance among monozygotic but not dizygotic twins

Environment

(a) Husband and wife with PBC (also shared same environment in childhood)

(b) Three unrelated females who shared same work environment developed PBC

(c) Women and daughter-in-law developed PBC

Combined/Undifferentiated Genes and Environment

(a) Two unrelated women sharing same environment as well as HLA and AMA patterns developed PBC

(b) Five of ten siblings in the same family developed PBC, two were asymptomatic but AMA positive, three AMA negative and asymptomatic

(c) Mother-daughter-close family friend developed PBC

A thorough list of studies reporting familial PBC in support of the role of genes and/or the environment in the pathogenesis of the disease is discussed in the main text, with the references in this table being a small representation.

compatible with PBC [10]. These figures are significantly higher than the general population, where $\mathrm{PBC}$ rates are between 0.7 and 7.5 cases per 100,000 [10]. This was also the case cited by Floreani et al. [11], who noted similar rates of $\mathrm{PBC}$ in families in Italy $(3.8 \%)$, and Tsuji et al. [12] cited a familial PBC prevalence of 5.1\%. Tsuji et al. [12] also noted a higher (944 times) PBC rate among families in Hiroshima, compared to 210 times higher in the remainder of Japan, when compared to the general population. Gershwin et al. [21] and Corpechot et al. [13] reported similar FDR incidence rates of PBC at 5.9\% and 4\%, respectively, which is consistent with other studies placing the familial incidence of $\mathrm{PBC}$ among FDR of PBC patients at approximately $4-6 \%[10-12,14]$. These rates are higher than those reported in an early study by Brind et al. [15] from King's College Hospital, which reports a familial incidence of $1.33 \%$. Jones et al. [14] notes a familial prevalence of PBC of $6.4 \%$, based on 157 patients with PBC, 10 of which had family histories of PBC ( 8 of the 10 were FDR). That study provided a clearer view of which relatives are most 
TABle 2: Main features of familial Primary Biliary Cirrhosis (PBC).

Prevalence of $\mathrm{PBC}$ is increased in families with members affected by PBC. Prevalence rates range from 4 to $6 \%$

Most affected members of the same family are females, with the most common pairing being in mother-daughters or sister-sisters

PBC rarely affects males, with few confirmed case reports of males relatives being affected

Increased incidence of autoimmune disease in families of PBC patients, most commonly Sicca syndrome and autoimmune thyroid disease

Diagnosis of a second or more family member often occurs within a short time interval of the initial case. This may be due to early screening for cholestasis or AMA initiated by the initial diagnosis

Concordance rates among monozygotic twins are among the highest of the autoimmune diseases

PBC in extended families (i.e., family members not related genetically) is rare, but has been reported mainly in husband and wife pairs, but at least one reports PBC in a female and her mother-in-law

Studies in support of the main findings in families of patients with PBC are discussed in the main text; AMA: antimitochondrial antibody.

at risk of developing PBC [14]. In general, FDR had a $0.72 \%$ prevalence of developing $\mathrm{PBC}$, whereas siblings of PBC patients had a prevalence of $0.4 \%$ [14]. Offspring of $\mathrm{PBC}$ patients had a much higher prevalence at $1.2 \%$, but daughters specifically had a $2.3 \%$ prevalence, which is consistent with clinical observation and case reports where mothers and daughters had the highest concordance of PBC [15], followed by sisters [21].

Numerous studies on the demographics and risk factors of $\mathrm{PBC}$ have demonstrated common features among larger cohorts of PBC patients and their families. A well-performed study by Gershwin et al. [21] demonstrated that autoimmune diseases were a common feature in $\mathrm{PBC}$, as it was present in $32 \%$ of $\mathrm{PBC}$ patients compared to $13 \%$ of controls. An increased incidence of autoimmune disease was also found in a study by Prince et al. [22], as well as in the study on PBC demographics by Corpechot and coworkers [13]. The French study by Corpechot et al. [13] indicates that autoimmune conditions were not only common among PBC patients, but also more common among their FDR. Bach and Schaffner also noted a higher incidence of autoimmune disease among FDR of PBC patients (approximately 71\%) [10].

\section{Primary Biliary Cirrhosis in Male Relatives}

Although rare in males, some cases of $\mathrm{PBC}$ occurring among male relatives have been reported. Early case reports have included PBC in a set of brothers [80]; however, it is uncertain whether this was indeed a case of familial PBC, as the diagnostic robustness in these early case reports is questionable. Tanaka et al. [81] cite two case studies of $\mathrm{PBC}$ occurring in brothers (one set in England, the other in France); however, the AMA status and clinical course of the two pairs remains obscure. Lazaridis et al. [16] report $\mathrm{PBC}$ occurring in brothers of $\mathrm{PBC}$ patients, but it has not been indicated whether the proband case was male or female. Of interest, the same investigators also report PBC cases occurring in father-daughter pairs, but none in father-son pairs [16]. Tanaka and coworkers also cite several father-daughter pairs and two brother-sister pairs, but again, clinical follow-up of these cases has not occurred, prohibiting a view of the disease progression and behaviour [81]. Lazaridis et al. found a PBC rate of $7.8 \%$ among brothers and $3.7 \%$ in fathers [16].

\section{Primary Biliary Cirrhosis in Mothers and Daughters}

As mentioned, the most frequent type of PBC clusters in families is reported in mother-daughter pairs [14, 15], followed by sisters [21] or mother-sister-daughter [17]. This is not surprising, as $\mathrm{PBC}$ is predominantly found in middle-aged females. Jaup and Zettergren [18] have reported a cluster of PBC in as many as four of six sisters. Abu-Mouch et al. [27] examined a cluster of PBC in a family in great detail. This cluster consisted of 10 siblings ( 8 sisters and 2 brothers) of Palestinian origin living in the same area. Five sisters presented with $\mathrm{PBC}$ within 10 years of each other and had high titre AMA with similar reactivity patterns and clinical stage [27]. Of the remaining three sisters, two tested weakly positive for AMA, and one had neither positive AMA nor $\mathrm{PBC}$ [27]. The two brothers and the mother were also negative for AMA and did not have findings suggestive of PBC.

Prevalence rates of $\mathrm{PBC}$ are increased among sisters and daughters of PBC patients. The study by Lazaridis et al. [16] found a $20.7 \%$ prevalence among sisters of PBC patients. In a report by Fagan et al., a mother-daughter pair, among the first-degree relatives, only the mother and daughter shared both genetic relations and a common environment, having been separated for only one year [17]. Environmental influences in the disease progression were noted due to the short period of time between the diagnosis of $\mathrm{PBC}$ in the two women [17]. Other early case reports also indicated a mother daughter pair being affected by PBC with quite a distinct clinical course amongst the affected individuals of the disease [19]. Douglas and Finlayson [28] also report PBC in a mother daughter pair, as well as in a close family friend. The daughter was the first of the three to present with PBC and was nursed through her terminal illness by the mother and family friend [28]. The mother and friend presented with PBC within 21 months of the daughter's death [28]. The short period of time between the diagnoses in the three women also seemed to indicate that these women were exposed for the same duration to a common environmental factor which triggered PBC. Autoantibody testing in the three women showed the presence of AMA [28]. Autoantibody testing of FDR of the mother-daughter pair revealed the presence of autoantibodies in several relatives, including AMA; however, none of these individuals were reported to have PBC [28]. It is possible that these individuals would have developed $\mathrm{PBC}$ or abnormal liver biochemistry, but no lengthy follow-up occurred. 
It is usually the case that at the time of diagnosis of PBC in a middle age woman, screening for AMA and a cholestatic form of abnormal liver function tests are also arranged for her siblings (mainly sisters) and mother. It is not unusual for some of those to turn positive for AMA with or without abnormal liver biochemistry. As mentioned, the mother daughter type of familial PBC is not the only one. Various other combinations have been noted but in most of those the diagnosis of $\mathrm{PBC}$ is difficult to prove and can be challenged [82]. In some of these, liver biopsies have not been performed or their findings are not entirely confirmatory of the disease, while in others the diagnosis of the disease is largely based on the presence of the antibodies and some nonspecific symptoms. No one has followed these asymptomatic AMA positive cases for long, but it is likely that a proportion of the AMA-positive asymptomatic relatives will develop abnormal liver biochemistry, and/or progress to PBC [48]. Due to the potential of a changing clinical course in asymptomatic patients, it is recommended that female FDR of PBC patients undergo immunological and liver function profiling, with a lengthy course of follow-up.

A higher frequency of monosomy of the $\mathrm{X}$ chromosome (a major X chromosome defect) in peripheral leukocytes of female PBC patients has been noted by Invernizzi and coworkers $[75,83]$. This characteristic has been found in other autoimmune diseases with a high female preponderance such as systemic sclerosis and autoimmune thyroid disease. Genes important for the homeostasis of sex hormones and the maintenance of immunological tolerance are located on the X chromosome. For example, the CD40 ligand $(C D 40 L)$ is an X-linked gene responsible for the X-linked immunodeficiency associated with increased serum levels of immunoglobulins $\mathrm{M}$; IgM levels are typically increased in the great majority of cases with PBC. It could be argued that $\mathrm{X}$ chromosome related dysregulation may explain the female preponderance of $\mathrm{PBC}$, and that the physiological inactivation of the $\mathrm{X}$ chromosome could account for the prevalence of PBC occurring peri-menopause [67, 83, 84]. This could also explain why systemic sclerosis and autoimmune thyroiditis are frequently seen in patients with PBC. The exact role of $\mathrm{X}$ monosomy in $\mathrm{PBC}$ may be more complex. Several scenarios have been proposed to explain how monosomy $\mathrm{X}$ is involved in the pathogenesis of $\mathrm{PBC}$ $[57,75,83-85]$. It has been argued [84] that "PBC could arise from a polygenic model with an X-linked major locus of susceptibility with genes escaping inactivation being involved; the female susceptibility might therefore as well derive from genes mapping on the $\mathrm{Y}$ chromosome [84]. The second scenario suggests that PBC susceptibility might arise from a multigenic complex inheritance model in which specific Y-linked genes are protective. Conversely, X-linked genes would interact with other genes and be involved via a dose-dependent effect" [84]. Subsequent studies supported the notion that enhanced $\mathrm{X}$ monosomy in peripheral blood mononuclear cells from patients with PBC preferentially involves only one parentally derived chromosome, and is not secondary to a constitutive nonrandom pattern of $\mathrm{X}$ chromosome inactivation [85]. The existence of X-chromosome inactivation in females offers a potential mechanism whereby
X-linked self-antigens may escape presentation in the thymus or in other peripheral sites that are involved in tolerance induction. The fact that X-chromosome monosomy in PBC is particularly evident in peripheral $\mathrm{B}$ lymphocytes and T lymphocytes in PBC patients' cell subpopulation which are important for the development of PBC, strengthens this notion. Thus, predisposed females may be in a state where under specific circumstances, they can express $\mathrm{X}$ linked autoantigens in the periphery to which they have been inefficiently tolerised. We need to identify these genes and dissect their exact relation with PBC. As monosomy $\mathrm{X}$ is not the characteristic feature of the peripheral blood mononuclear cells from all patients with PBC, it can be argued that inactivation of chromosome X cannot explain $a$ priori the induction of PBC.

\section{Twin Studies}

Few studies or case reports exist that specifically examine $\mathrm{PBC}$ in twins. Chohan reports a set of twin sisters with $\mathrm{PBC}$, but genetic, environmental, and other relevant medical histories are lacking in this report [86]. Selmi et al. [26] performed a study consisting of 8 sets each of monozygotic and dizygotic pairs. Four pairs were females, and the remaining four were male-female. The concordance rate of $\mathrm{PBC}$ among the monozygotic pairs was 0.63 (5 of 8 pairs were concordant for PBC) [86]. It could be argued that as the concordance was not 1.0, genetic factors do not play a role in PBC pathogenesis. However, a concordance of 0.63 is one of the highest among the autoimmune diseases. As well, it is possible that the asymptomatic twins did eventually develop PBC over time. All affected individuals were female, with the majority (four of five sets) being of white ethnicity, and one set being Asian [26]. The age of PBC presentation was between 33 and 60 years [26]. Most of the sets presented at the same age, or within two years of each other. Most twin sets had the same presenting symptoms of pruritus, fatigue, or a combination of the two. In one pair (set 2), one twin presented with liver failure and required liver transplantation, whereas the other twin had no presenting symptoms over a 13-year follow-up [26].

Among the monozygotic twin sets, three were discordant for PBC. Two of them were positive for AMA with no abnormal liver function tests. Most of the twin sets had Sicca symptomatology, which is present in the majority of PBC patients. However, set 4 had autoimmune hypothyroidism, and one twin in set 1 had autoimmune hepatitis [26]. The high incidence of other autoimmune diseases indicates a possible propensity to autoimmunity in genetically susceptible individuals. The differing histological grades, dissimilar clinical course, and variable autoimmunity do not favour a clear cut genetic cause. Indeed, there was great variability among the sets of twins. Twins in set 4 had the same age of presentation as well as presenting symptoms, histological grades, and autoimmune hypothyroid [26]. Set 2 had few similarities, and drastically different clinical courses and histological grades. Selmi et al. [26] indicates that these variances may be due to an interplay between genes and environment, as well as possible differences on the epigenetic 
level. Although their study was unable to indicate specific environmental triggers, other authors have suggested environmental toxins, xenobiotics, and microbial/self molecular mimicry [56-60, 65, 66, 70, 71, 73-75, 77, 78, 87-102].

\section{PBC in the Extended Family}

Twin studies have provided evidence towards both genetic and environmental influences in the pathogenesis of PBC. Extended families provide some evidence towards environmental influences, as individuals such as husbands and wives share a common environment, but usually no genetic similarities. The report by Abu-Mouch has provided examples of such cases [27]. One cluster was a husband and wife with $\mathrm{PBC}$, who grew up in the same neighbourhood in Michigan, and who's only reported risk factors were cigarette smoke, and recurrent UTI's - a well-documented risk factor for PBC-in the female. The husband was five years younger and developed the disease five years after his wife had been diagnosed with $\mathrm{PBC}$, but he had histologically more advanced disease compared to his wife [27]. The lack of husband/wife PBC pairs in the literature clearly indicates the extreme rarity of conjugal $\mathrm{PBC}$. Another cluster was a family where $\mathrm{PBC}$ was present in two genetically unrelated individuals (grandmother and her daughter-in-law) [27]. The daughter and grand-daughter of the grandmother were AMA positive but asymptomatic, and the daughter-in-law was from Korea (where there is a low PBC rate). The grandmother and her daughter-in-law shared the same house for 2 years and then lived in the same neighbourhood for 30 years [27]. Whether or not environment played a large role in that cluster, or whether those individuals had genetic susceptibility to $\mathrm{PBC}$ and the development of the disease was purely by chance, remains unknown.

\section{Genetic and Epigenetic Studies}

A point of interest noted above is that which relates to the increased prevalence of autoimmune diseases unrelated to $\mathrm{PBC}$ or other autoimmune diseases. The exact cause of this may relate to genes conferring susceptibility to various autoimmune diseases. For example, Hemminki et al. [103] indicate relatively weak but significant associations between STAT4 and CTLA4 loci with RA and SLE in the case of STAT4, and to RA and other autoimmune disease in the case of CTLA4. Hirschfield et al. [25] set out to identify genetic loci conferring a risk for $\mathrm{PBC}$, by carrying out a genome-wide association analysis of $536 \mathrm{PBC}$ patients and 1536 controls from Canada and the USA. A significant association between PBC and 13 loci across the HLA class II region, with HLA DQB1 having the strongest association [25]. Associations were also made in non-HLA genes, with two single-nucleotide polymorphisms (SNPs) at the IL12A loci, and one SNP at the IL12RB2 locus. Associations with SNPs at the STAT4 and CTLA4 loci were also found, but to a lesser degree [25]. Liu et al. [23] had similar findings in regards to IL12A and IL12RB in an Italian cohort, in addition to associations with several HLA regions (namely, DRB1,
DQA1, DQB1, DQA2), IRF5, SPIB, and the IKZF3-ORMDL3 of chromosome 17q12-21. These findings are interesting, as STAT4 plays a role in IL12 signalling, and its role in PBC may be significant due to its association with other autoimmune diseases such as RA and SLE [23]. Based on these findings, it would be expected that $\mathrm{PBC}$ would co-occur frequently with RA or SLE, and vice versa. However, PBC more often co-occurs with Sicca syndrome and autoimmune thyroiditis, and we would expect that these diseases also share an association with STAT4. Hirschfield et al. [24] have also demonstrated an association between $\mathrm{PBC}$ and genes at the IRF5-TNPO3 locus, at chromosome 17q12-21, and at loci MMEL1 in a cohort of PBC patients of European descent. These findings were of great interest, as IRF5-TNPO3 has been previously associated with other autoimmune diseases, namely, SLE, systemic sclerosis, and Sjögrens syndrome [24]. Hirschfield's study has also provided fascinating data in regards to a potential genetic characterisation of individuals with ANA positivity [24]. Within the main cohort, 462 $\mathrm{PBC}$ individuals with ANA positivity were evaluated as a subset, and an association was found with HLA-DPB1 in those with anti-Sp100 [24]. No association was found in those with ANA positivity with anti-gp210 [24]. Although this cohort was relatively small, the findings indicate a potential correlation between HLA type and ANA status, which warrants further study due to its potential prognostic value.

Several HLA haplotypes have been reported within families, but only HLA-DR8 has been reported to be found consistently. Tsuji et al. [12] analysed 18 patients from 8 families with more than one FDR with PBC. Affected members among the same family shared HLA haplotypes, but these patterns were not often shared among the 8 families, with the exception of HLA-DR8, which was found in $29.4 \%$ of patients [12]. HLA DR8 has been considered a risk factor for developing PBC, especially in Caucasian populations [104]. Von Mach [20] notes similar antigenic and HLA patterns in a report of two women who were not related, but shared a common environment (they shared a desk at school until the age of 10). Both women had a 25 -year history of liver enzyme abnormalities and were diagnosed with $\mathrm{PBC}$ in a short time interval between each other [20]. Both were AMA positive, with a strong reaction to PDC-E2 [20]. Both women shared HLA haplotypes A2, B51, DR4, DR53, and, not surprisingly, DR8 [20, 104].

The differing concordance rates among monozygotic twins noted above may be attributed to epigenetic causes. Disconcordance due to epigenetic factors among identical twins has been observed in other conditions. In BeckwithWiedmann syndrome, differences in imprinting of the KCNQ10T1 gene have been implicated in the discordance rates among twins with this condition. As well, methylation pattern differences among twins with Silver-Russell syndrome have also been demonstrated to account for discordance among monozygotic twins. A recent study by Mitchell et al. [105] reports epigenetic differences found in discordant monozygotic twins with PBC. It has been found that there is an increased $\mathrm{X}$ chromosome monosomy rate in the peripheral lymphocytes of female patients with $\mathrm{PBC}$ 
$[75,83,105]$. Most genes on the inactivated X chromosome are silenced via promoter methylation, but a small proportion of these may escape this process. Mitchell et al. [105] obtained mRNA and DNA from peripheral blood from four sets of monozygotic twins who were discordant for PBC. Two genes, CLIC2 and PIN4, were consistently downregulated in the twin with PBC [105]. This was not found in the healthy twin or in control subjects. In both genes, it was found that methylation was partial or variable and did not predict transcript levels or X inactivation status [105]. CLIC2 encodes for an intracellular calcium channel and regulates calcium homeostasis via the ryanodine receptors. Abnormalities in intracellular calcium may lead to cellular and tissue damage which, if occurring in the liver, may lead to liver disease [105]. PIN4 is involved in mitoses, cell proliferation, chromatin remodelling, and epigenetic mechanisms involving ATP-dependent changes to histone modifications [105]. PIN4 dysregulation could therefore potentially lead to an alteration in the function of genes which may be protective or pathogenic [105]. What role these genes play in the pathogenesis of PBC and its variable concordance among identical twins remain to be seen [67, 78]. Epigenetic studies of related and nonrelated (but sharing a common environment) individuals with PBC also warrant further study.

\section{Future Prospects}

Areas for future work include elucidating those environmental factors which are associated with PBC, and determining the mechanism by which they induce disease. This is not, however, an easy task. Most of the work done in the search for environmental triggers rely on animal models, addressing one compound at the time. Worryingly enough, the most frequent tool that epidemiologists can use in order to identify multiple environmental exposures linked to a specific autoimmune disease (PBC in our case) is that of questionnaires or access to data extracted from medical records. This is in sharp contrast to the information of large genome-wide association studies which more or less provide measurable information for the totality of genes as risk factors. Ideally, one would like to be able to accurately measure simultaneously the totality of exposures received by a person during life in order to investigate the environmental causes of chronic diseases. These factors may also include the chemical by-products within the body, which are produced by inflammation, oxidative stress, gut flora and infection, as well as through a bio-psycho-social axis. The collation of all these external and internal environmental factors from conception onwards has been named as the "exposome" [106-108]. It has been suggested that several measurements on exposomal factors may be done through multiple blood samples over several years, which would characterise changes in environment [108]. In the case of familial PBC, multiple samples of at risk individuals may shed some light on which factors are critical for the development or progression of $\mathrm{PBC}$. The exposome may very well turn out to be the environmental equivalent of the genome-wide association study, which would allow a quantitative measure of the effects of a broad range of environmental triggers and carefully selected infectious agents in the pathogenesis of disease [109].

\section{Conclusions}

In conclusion, studies of patients with $\mathrm{PBC}$ demonstrate a higher prevalence of $\mathrm{PBC}$ among families, which is too high and too frequent to be attributed to chance alone. As well, several studies have shown that unrelated individuals who shared a common environment also had a higher incidence of PBC. As these reports are rare, it is difficult to speculate as to whether these are attributable to chance, or a common environmental trigger. Nevertheless, each of these studies has a story to tell. Familial PBC raises the question as to whether genetic or environmental factors are at the cause of the disease, and twin studies raise the possibility of epigenetic phenomena also. It most likely that genetic, epigenetic, and environmental factors work in unison, with varying degrees of influence on the disease progression within the individual as well as their families. Despite our advances in knowledge surrounding these individual factors, it will likely be some time before the causes of PBC and their interaction with one another are well defined.

\section{Abbreviations}

AMA: Antimitochondrial Antibodies

ANA: Antinuclear antibodies

BCOADC: Branched chain oxo-acid dehydrogenase complex

E3BP: $\quad$ E3 binding protein

FDR: $\quad$ First-degree relatives

PBC: $\quad$ Primary Biliary Cirrhosis

PDC: $\quad$ Pyruvate dehydrogenase complex

OADC: Oxo-acid dehydrogenase complex

OGDC: Oxoglutarate dehydrogenase complex.

\section{Authors' Contribution}

Eirini I. Rigopoulou and Dimitrios P. Bogdanos equally contributed.

\section{References}

[1] M. M. Kaplan and M. E. Gershwin, "Primary biliary cirrhosis," New England Journal of Medicine, vol. 353, no. 12, pp. 1261-1273, 2005.

[2] S. Hohenester, R. P. J. Oude-Elferink, and U. Beuers, "Primary biliary cirrhosis," Seminars in Immunopathology, vol. 31, no. 3, pp. 283-307, 2009.

[3] J. Neuberger, "Primary biliary cirrhosis," Lancet, vol. 350, no. 9081, pp. 875-879, 1997.

[4] O. E. W. James, R. Bhopal, D. Howel, J. Gray, A. D. Burt, and J. V. Metcalf, "Primary biliary cirrhosis once rare, now common in the United Kingdom?" Hepatology, vol. 30, no. 2, pp. 390-394, 1999.

[5] S. Sood, P. J. Gow, J. M. Christie, and P. W. Angus, "Epidemiology of primary biliary cirrhosis in Victoria, Australia: high prevalence in migrant populations," Gastroenterology, vol. 127, no. 2, pp. 470-475, 2004. 
[6] W. R. Kim, K. D. Lindor, G. R. Locke et al., "Epidemiology and natural history of primary biliary cirrhosis in a U.S. community," Gastroenterology, vol. 119, no. 6, pp. 16311636, 2000.

[7] M. G. Peters, A. M. Di Bisceglie, K. V. Kowdley et al., "Differences between Caucasian, African American, and Hispanic patients with primary biliary cirrhosis in the United States," Hepatology, vol. 46, no. 3, pp. 769-775, 2007.

[8] R. G. P. Watson, P. W. Angus, M. Dewar, B. Goss, R. B. Sewell, and R. A. Smallwood, "Low prevalence of primary biliary cirrhosis in Victoria, Australia," Gut, vol. 36, no. 6, pp. 927930, 1995.

[9] A. C. Anand, E. Elias, and J. M. Neuberger, "End-stage primary biliary cirrhosis in a first generation migrant south Asian population," European Journal of Gastroenterology and Hepatology, vol. 8, no. 7, pp. 663-666, 1996.

[10] N. Bach and F. Schaffner, "Familial primary biliary cirrhosis," Journal of Hepatology, vol. 20, no. 6, pp. 698-701, 1994.

[11] A. Floreani, R. Naccarato, and M. Chiaramonte, "Prevalence of familial disease in primary biliary cirrhosis in Italy," Journal of Hepatology, vol. 26, no. 3, pp. 737-738, 1997.

[12] K. Tsuji, Y. Watanabe, J. Van De Water et al., "Familial primary biliary cirrhosis in Hiroshima," Journal of Autoimmunity, vol. 13, no. 1, pp. 171-178, 1999.

[13] C. Corpechot, Y. Chrétien, O. Chazouillères, and R. Poupon, "Demographic, lifestyle, medical and familial factors associated with primary biliary cirrhosis," Journal of Hepatology, vol. 53, no. 1, pp. 162-169, 2010.

[14] D. E. J. Jones, F. E. Watt, J. V. Metcalf, M. F. Bassendine, and O. F. W. James, "Familial primary hiliary cirrhosis reassessed: a geographically-based population study," Journal of Hepatology, vol. 30, no. 3, pp. 402-407, 1999.

[15] A. M. Brind, G. P. Bray, B. C. Portmann, and R. Williams, "Prevalence and pattern of familial disease in primary biliary cirrhosis,” Gut, vol. 36, no. 4, pp. 615-617, 1995.

[16] K. N. Lazaridis, B. D. Juran, G. M. Boe et al., "Increased prevalence of antimitochondrial antibodies in first-degree relatives of patients with primary biliary cirrhosis," Hepatology, vol. 46, no. 3, pp. 785-792, 2007.

[17] E. Fagan, R. Williams, and S. Cox, "Primary biliary cirrhosis in mother and daughter," British Medical Journal, vol. 2, no. 6096, p. 1195, 1977.

[18] B. H. Jaup and L. S. W. Zettergren, "Familial occurrence of primary biliary cirrhosis associated with hypergammaglobulinemia in descendants: a family study," Gastroenterology, vol. 78, no. 3, pp. 549-555, 1980.

[19] M. J. Tong, K. M. Nies, T. B. Reynolds, and F. P. Quismorio, "Immunological studies in familial primary biliary cirrhosis," Gastroenterology, vol. 71, no. 2, pp. 305-307, 1976.

[20] M. von Mach, "Primary biliary cirrhosis in classmates: coincidence of enigmatic environmental influence?" EXCLI, vol. 1, pp. 1-7, 2002.

[21] M. E. Gershwin, C. Selmi, H. J. Worman et al., "Risk factors and comorbidities in primary biliary cirrhosis: a controlled interview-based study of 1032 patients," Hepatology, vol. 42, no. 5, pp. 1194-1202, 2005.

[22] M. I. Prince, S. J. Ducker, and O. F. W. James, "Case-control studies of risk factors for primary biliary cirrhosis in two United Kingdom populations," Gut, vol. 59, no. 4, pp. 508512, 2010.

[23] X. Liu, P. Invernizzi, Y. Lu et al., "Genome-wide metaanalyses identify three loci associated with primary biliary cirrhosis," Nature Genetics, vol. 42, no. 8, pp. 658-660, 2010.
[24] G. M. Hirschfield, X. Liu, Y. Han et al., "Variants at IRF5TNPO3, 17q12-21 and MMEL1 are associated with primary biliary cirrhosis," Nature Genetics, vol. 42, no. 8, pp. 655-657, 2010.

[25] G. M. Hirschfield, X. Liu, C. Xu et al., "Primary biliary cirrhosis associated with HLA, IL12A, and IL12RB2 variants," New England Journal of Medicine, vol. 360, no. 24, pp. 25442555, 2009.

[26] C. Selmi, M. J. Mayo, N. Bach et al., "Primary biliary cirrhosis in monozygotic and dizygotic twins: genetics, epigenetics, and environment," Gastroenterology, vol. 127, no. 2, pp. 485492, 2004.

[27] S. Abu-Mouch, C. Selmi, G. D. Benson et al., "Geographic clusters of primary biliary cirrhosis," Clinical and Developmental Immunology, vol. 10, no. 2-4, pp. 127-131, 2003.

[28] J. G. Douglas and N. D. C. Finlayson, "Are increased individual susceptibility and environmental factors both necessary for the development of primary biliary cirrhosis?" British Medical Journal, vol. 2, no. 6187, pp. 419-420, 1979.

[29] D. P. Bogdanos, H. Baum, and D. Vergani, "Antimitochondrial and other autoantibodies," Clinics in Liver Disease, vol. 7, no. 4, pp. 759-777, 2003.

[30] D. P. Bogdanos, P. Invernizzi, I. R. Mackay, and D. Vergani, "Autoimmune liver serology: current diagnostic and clinical challenges," World Journal of Gastroenterology, vol. 14, no. 21, pp. 3374-3387, 2008.

[31] J. C. Courvalin and H. J. Worman, "Nuclear envelope protein autoantibodies in primary biliary cirrhosis," Seminars in Liver Disease, vol. 17, no. 1, pp. 79-90, 1997.

[32] C. Dähnrich, A. Pares, L. Caballeria et al., "New ELISA for detecting primary biliary cirrhosis-specific antimitochondrial antibodies," Clinical Chemistry, vol. 55, no. 5, pp. 978985, 2009.

[33] H. Liu, G. L. Norman, Z. Shums et al., "PBC Screen: an IgG/IgA dual isotype ELISA detecting multiple mitochondrial and nuclear autoantibodies specific for primary biliary cirrhosis," Journal of Autoimmunity, vol. 35, no. 4, pp. 436442, 2010.

[34] D. P. Bogdanos and L. Komorowski, "Disease-specific autoantibodies in primary biliary cirrhosis," Clinica Chimica Acta, vol. 412, no. 7-8, pp. 502-512, 2011.

[35] M. D. Van Norstrand, M. Malinchoc, K. D. Lindor et al., "Quantitative measurement of autoantibodies to recombinant mitochondrial antigens in patients with primary biliary cirrhosis: relationship of levels of autoantibodies to disease progression," Hepatology, vol. 25, no. 1, pp. 6-11, 1997.

[36] E. I. Rigopoulou, D. P. Bogdanos, C. Liaskos et al., "Antimitochondrial antibody immunofluorescent titres correlate with the number and intensity of immunoblot-detected mitochondrial bands in patients with primary biliary cirrhosis," Clinica Chimica Acta, vol. 380, no. 1-2, pp. 118-121, 2007.

[37] E. I. Rigopoulou, E. T. Davies, D. P. Bogdanos et al., "Antimitochondrial antibodies of immunoglobulin G3 subclass are associated with a more severe disease course in primary biliary cirrhosis," Liver International, vol. 27, no. 9, pp. 12261231, 2007.

[38] E. I. Rigopoulou, E. T. Davies, A. Pares et al., "Prevalence and clinical significance of isotype specific antinuclear antibodies in primary biliary cirrhosis," Gut, vol. 54, no. 4, pp. 528-532, 2005. 
[39] K. Miyachi, R. W. Hankins, H. Matsushima et al., "Profile and clinical significance of anti-nuclear envelope antibodies found in patients with primary biliary cirrhosis: a multicenter study," Journal of Autoimmunity, vol. 20, no. 3, pp. 247-254, 2003.

[40] M. Nakamura, H. Kondo, T. Mori et al., "Anti-gp210 and anti-centromere antibodies are different risk factors for the progression of primary biliary cirrhosis," Hepatology, vol. 45, no. 1, pp. 118-127, 2007.

[41] D. P. Bogdanos, C. Liaskos, A. Pares et al., "Anti-gp210 antibody mirrors disease severity in primary biliary cirrhosis," Hepatology, vol. 45, no. 6, p. 1583, 2007.

[42] D.-P. Bogdanos, C. Liaskos, E. I. Rigopoulou, and G. N. Dalekos, "Anti-mitochondrial antibodies in patients with systemic lupus erythematosus: revealing the unforeseen," Clinica Chimica Acta, vol. 373, no. 1-2, pp. 183-184, 2006.

[43] D.-P. Bogdanos, A. Pares, J. Rodés et al., "Primary biliary cirrhosis specific antinuclear antibodies in patients from Spain," American Journal of Gastroenterology, vol. 99, no. 4, pp. 763-765, 2004.

[44] D.-P. Bogdanos, D. Vergani, P. Muratori, L. Muratori, and F. B. Bianchi, "Specificity of anti-sp100 antibody for primary biliary cirrhosis," Scandinavian Journal of Gastroenterology, vol. 39, no. 4, pp. 405-406, 2004.

[45] P. Invernizzi, M. Podda, P. M. Battezzati et al., "Autoantibodies against nuclear pore complexes are associated with more active and severe liver disease in primary biliary cirrhosis," Journal of Hepatology, vol. 34, no. 3, pp. 366-372, 2001.

[46] S. Oertelt, R. Rieger, C. Selmi et al., "A sensitive bead assay for antimitochondrial antibodies: chipping away at AMAnegative primary biliary cirrhosis," Hepatology, vol. 45, no. 3, pp. 659-665, 2007.

[47] D. Vergani and D. P. Bogdanos, "Positive markers in AMAnegative PBC," American Journal of Gastroenterology, vol. 98, no. 2, pp. 241-243, 2003.

[48] J. V. Metcalf, H. C. Mitchison, J. M. Palmer, D. E. Jones, M. F. Bassendine, and O. F. W. James, "Natural history of early primary biliary cirrhosis," Lancet, vol. 348, no. 9039, pp. 1399-1402, 1996.

[49] H. C. Mitchison, M. F. Bassendine, and A. Hendrick, "Positive antimitochondrial antibody but normal alkaline phosphatase: is this primary biliary cirrhosis?" Hepatology, vol. 6, no. 6, pp. 1279-1284, 1986.

[50] J. V. Metcalf, R. S. Bhopal, J. Gray, D. Howel, and O. F. W. James, "Incidence and prevalence of primary biliary cirrhosis in the city of Newcastle upon Tyne, England," International Journal of Epidemiology, vol. 26, no. 4, pp. 830-836, 1997.

[51] K. E. Kisand, K. V. Kisand, A. L. Karvonen et al., "Antibodies to pyruvate dehydrogenase in primary biliary cirrhosis: correlation with histology," APMIS, vol. 106, no. 9, pp. 884-892, 1998.

[52] K. D. Lindor, M. E. Gershwin, R. Poupon, M. Kaplan, N. V. Bergasa, and E. J. Heathcote, "Primary biliary cirrhosis," Hepatology, vol. 50, no. 1, pp. 291-308, 2009.

[53] C. Szostecki, H. H. Guldner, and H. Will, "Autoantibodies against "nuclear dots" in primary biliary cirrhosis," Seminars in Liver Disease, vol. 17, no. 1, pp. 71-78, 1997.

[54] J. Wesierska-Gadek, E. Penner, P. M. Battezzati et al., "Correlation of initial autoantibody profile and clinical outcome in primary biliary cirrhosis," Hepatology, vol. 43, no. 5, pp. 1135-1144, 2006.
[55] W. H. Yang, J. H. Yu, A. Nakajima, D. Neuberg, K. Lindor, and D. B. Bloch, "Do antinuclear antibodies in primary biliary cirrhosis patients identify increased risk for liver failure?" Clinical Gastroenterology and Hepatology, vol. 2, no. 12, pp. 1116-1122, 2004.

[56] M. E. Gershwin and I. R. Mackay, "Primary biliary cirrhosis: paradigm or paradox for autoimmunity," Gastroenterology, vol. 100, no. 3, pp. 822-833, 1991.

[57] M. E. Gershwin and I. R. Mackay, "The causes of primary biliary cirrhosis: convenient and inconvenient truths," Hepatology, vol. 47, no. 2, pp. 737-745, 2008.

[58] I. R. Mackay, S. Whittingham, S. Fida et al., "The peculiar autoimmunity of primary biliary cirrhosis," Immunological Reviews, vol. 174, pp. 226-237, 2000.

[59] D. P. Bogdanos and D. Vergani, "Origin of cross-reactive autoimmunity in primary biliary cirrhosis," Liver International, vol. 26, no. 6, pp. 633-635, 2006.

[60] D. P. Bogdanos and D. Vergani, "Bacteria and primary biliary cirrhosis," Clinical Reviews in Allergy and Immunology, vol. 36, no. 1, pp. 30-39, 2009.

[61] D. P. Bogdanos, H. Baum, D. Vergani, and A. K. Burroughs, "The role of E. coli infection in the pathogenesis of primary biliary cirrhosis," Disease Markers, vol. 29, no. 6, pp. 301-311, 2010.

[62] S. Shimoda, M. Nakamura, H. Ishibashi, K. Hayashida, and Y. Niho, "HLA DRB4 0101-restricted immunodominant $\mathrm{T}$ cell autoepitope of pyruvate dehydrogenase complex in primary biliary cirrhosis: evidence of molecular mimicry in human autoimmune diseases," Journal of Experimental Medicine, vol. 181, no. 5, pp. 1835-1845, 1995.

[63] S. Shimoda, M. Nakamura, H. Shigematsu et al., "Mimi-cry peptides of human PDC-E2 163-176 peptide, the immunodominant T- cell epitope of primary biliary cirrhosis," Hepatology, vol. 31, no. 6, pp. 1212-1216, 2000.

[64] S. Shimoda, J. Van De Water, A. Ansari et al., "Identification and precursor frequency analysis of a common T cell epitope motif in mitochondrial autoantigens in primary biliary cirrhosis," Journal of Clinical Investigation, vol. 102, no. 10, pp. 1831-1840, 1998.

[65] D. E. J. Jones, “Enesis of primary biliary cirrhosis," Gut, vol. 56, no. 11, pp. 1615-1624, 2007.

[66] C. Selmi and M. E. Gershwin, "The role of environmental factors in primary biliary cirrhosis," Trends in Immunology, vol. 30, no. 8, pp. 415-420, 2009.

[67] C. Selmi, P. Invernizzi, M. Zuin, M. Podda, M. F. Seldin, and M. E. Gershwin, "Genes and (auto)immunity in primary biliary cirrhosis," Genes and Immunity, vol. 6, no. 7, pp. 543-556, 2005.

[68] R. J. Q. McNally, S. Ducker, and O. F. W. James, "Are transient environmental agents involved in the cause of primary biliary cirrhosis? Evidence from space-time clustering analysis," Hepatology, vol. 50, no. 4, pp. 1169-1174, 2009.

[69] D. Alvaro, P. Invernizzi, P. Onori et al., "Estrogen receptors in cholangiocytes and the progression of primary biliary cirrhosis," Journal of Hepatology, vol. 41, no. 6, pp. 905-912, 2004.

[70] K. Amano, P. S. C. Leung, R. Rieger et al., "Chemical xenobiotics and mitochondrial autoantigens in primary biliary cirrhosis: identification of antibodies against a common environmental, cosmetic, and food additive, 2-octynoic acid," Journal of Immunology, vol. 174, no. 9, pp. 5874-5883, 2005. 
[71] K. Amano, P. S. C. Leung, Q. Xu et al., "Xenobioticinduced loss of tolerance in rabbits to the mitochondrial autoantigen of primary biliary cirrhosis is reversible," Journal of Immunology, vol. 172, no. 10, pp. 6444-6452, 2004.

[72] D. Bogdanos, T. Pusl, C. Rust, D. Vergani, and U. Beuers, "Primary biliary cirrhosis following lactobacillus vaccination for recurrent vaginitis," Journal of Hepatology, vol. 49, no. 3, pp. 466-473, 2008.

[73] D. P. Bogdanos, H. Baum, M. Okamoto et al., "Primary biliary cirrhosis is characterized by IgG3 antibodies crossreactive with the major mitochondrial autoepitope and its lactobacillus mimic," Hepatology, vol. 42, no. 2, pp. 458-465, 2005.

[74] D. P. Bogdanos, H. Baum, U. C. Sharma et al., "Antibodies against homologous microbial caseinolytic proteases $\mathrm{P}$ characterise primary biliary cirrhosis," Journal of Hepatology, vol. 36, no. 1, pp. 14-21, 2002.

[75] P. Invernizzi, M. Miozzo, P. M. Battezzati et al., "Frequency of monosomy $\mathrm{X}$ in women with primary biliary cirrhosis," Lancet, vol. 363, no. 9408, pp. 533-535, 2004.

[76] D. E. J. Jones and P. T. Donaldson, "Genetic factors in the pathogenesis of primary biliary cirrhosis," Clinics in Liver Disease, vol. 7, no. 4, pp. 841-864, 2003.

[77] D. Vergani, D. P. Bogdanos, and H. Baum, "Unusual suspects in primary biliary cirrhosis," Hepatology, vol. 39, no. 1, pp. 38-41, 2004.

[78] C. Selmi, P. Invernizzi, M. Zuin, M. Podda, and M. E. Gershwin, "Genetics and geoepidemiology of primary biliary cirrhosis: following the footprints to disease etiology," Seminars in Liver Disease, vol. 25, no. 3, pp. 265-280, 2005.

[79] D. Smyk, M. G. Mytilinaiou, E. I. Rigopoulou, and D. P. Bogdanos, "PBC triggers in water reservoirs, coal mining areas and waste disposal sites: from Newcastle to New York," Disease Markers, vol. 29, no. 6, pp. 337-344, 2010.

[80] R. Bown, M. L. Clark, and D. Doniach, "Primary biliary cirrhosis in brothers," Postgraduate Medical Journal, vol. 51, no. 592, pp. 110-115, 1975.

[81] A. Tanaka, A. T. Borchers, H. Ishibashi, A. A. Ansari, C. L. Keen, and M. E. Gershwin, "Genetic and familial considerations of primary biliary cirrhosis," American Journal of Gastroenterology, vol. 96, no. 1, pp. 8-15, 2001.

[82] Y. Kato, K. Suzuki, and M. Kumagai, "Familial primary biliary cirrhosis. Immunological and genetic study," American Journal of Gastroenterology, vol. 75, no. 3, pp. 188-191, 1981.

[83] P. Invernizzi, M. Miozzo, C. Selmi et al., "X chromosome monosomy: a common mechanism for autoimmune diseases," Journal of Immunology, vol. 175, no. 1, pp. 575-578, 2005.

[84] C. Selmi, P. Invernizzi, M. Miozzo, M. Podda, and M. E. Gershwin, "Primary biliary cirrhosis: does X mark the spot?" Autoimmunity Reviews, vol. 3, no. 7-8, pp. 493-499, 2004.

[85] M. Miozzo, C. Selmi, B. Gentilin et al., "Preferential X chromosome loss but random inactivation characterize primary biliary cirrhosis," Hepatology, vol. 46, no. 2, pp. 456-462, 2007.

[86] M. R. Chohan, "Primary biliary cirrhosis in twin sisters," Gut, vol. 14, no. 3, pp. 213-214, 1973.

[87] C. Selmi, D. L. Balkwill, P. Invernizzi et al., "Patients with primary biliary cirrhosis react against a ubiquitous xenobiotic-metabolizing bacterium," Hepatology, vol. 38, no. 5, pp. 1250-1257, 2003.
[88] D. P. Bogdanos, H. Baum, P. Butler et al., "Association between the primary biliary cirrhosis specific anti-sp100 antibodies and recurrent urinary tract infection," Digestive and Liver Disease, vol. 35, no. 11, pp. 801-805, 2003.

[89] D. P. Bogdanos, H. Baum, A. Grasso et al., "Microbial mimics are major targets of crossreactivity with human pyruvate dehydrogenase in primary biliary cirrhosis," Journal of Hepatology, vol. 40, no. 1, pp. 31-39, 2004.

[90] D. P. Bogdanos, H. Baum, F. Gunsar et al., "Extensive homology between the major immunodominant mitochondrial antigen in primary biliary cirrhosis and Helicobacter pylori does not lead to immunological crossreactivity," Scandinavian Journal of Gastroenterology, vol. 39, no. 10, pp. 981-987, 2004.

[91] D. P. Bogdanos, K. Choudhuri, and D. Vergani, "Molecular mimicry and autoimmune liver disease: virtuous intentions, malign consequences," Liver, vol. 21, no. 4, pp. 225-232, 2001.

[92] D. P. Bogdanos, A. Koutsoumpas, H. Baum, and D. Vergani, "Borrelia Burgdorferi: a new self-mimicking trigger in primary biliary cirrhosis," Digestive and Liver Disease, vol. 38, no. 10, pp. 781-782, 2006.

[93] D. P. Bogdanos, A. Pares, H. Baum et al., "Disease-specific cross-reactivity between mimicking peptides of heat shock protein of mycobacterium gordonae and dominant epitope of E2 subunit of pyruvate dehydrogenase is common in Spanish but not British patients with primary biliary cirrhosis," Journal of Autoimmunity, vol. 22, no. 4, pp. 353-362, 2004.

[94] A. K. Burroughs, P. Butler, M. J. E. Sternberg, and H. Baum, "Molecular mimicry in liver disease," Nature, vol. 358, no. 6385, pp. 377-378, 1992.

[95] P. Butler, J. Hamilton-Miller, H. Baum, and A. K. Burroughs, "Detection of M2 antibodies in patients with recurrent urinary tract infection using an ELISA and purified PBC specific antigens. Evidence for a molecular mimicry mechanism in the pathogenesis of primary biliary cirrhosis?" Biochemistry and Molecular Biology International, vol. 35, no. 3, pp. 473-485, 1995.

[96] D. Vergani, F. Alvarez, F. B. Bianchi et al., "Liver autoimmune serology: a consensus statement from the committee for autoimmune serology of the International Autoimmune Hepatitis Group," Journal of Hepatology, vol. 41, no. 4, pp. 677-683, 2004.

[97] A. S. Abdulkarim, L. M. Petrovic, W. R. Kim, P. Angulo, R. V. Lloyd, and K. D. Lindor, "Primary biliary cirrhosis: an infectious disease caused by Chlamydia pneumoniae?" Journal of Hepatology, vol. 40, no. 3, pp. 380-384, 2004.

[98] A. Koutsoumpas, M. Mytilinaiou, D. Polymeros, G. N. Dalekos, and D. P. Bogdanos, "Anti-Helicobacter pylori antibody responses specific for VacA do not trigger primary biliary cirrhosis-specific antimitochondrial antibodies," European Journal of Gastroenterology and Hepatology, vol. 21, no. 10, p. 1220, 2009.

[99] P. S. C. Leung, O. Park, S. Matsumura, A. A. Ansari, R. L. Coppel, and M. E. Gershwin, "Is there a relation between Chlamydia infection and primary biliary cirrhosis?" Clinical and Developmental Immunology, vol. 10, no. 2-4, pp. 227-233, 2003.

[100] A. Mason, L. Xu, Z. Shen et al., "Patients with primary biliary cirrhosis make anti-viral and anti-mitochondrial antibodies to mouse mammary tumor virus," Gastroenterology, vol. 127, no. 6, pp. 1863-1865, 2004. 
[101] L. Xu, Z. Shen, L. Guo et al., "Does a betaretrovirus infection trigger primary biliary cirrhosis?" Proceedings of the National Academy of Sciences of the United States of America, vol. 100, no. 14, pp. 8454-8459, 2003.

[102] J. Van de Water, H. Ishibashi, R. L. Coppel, and M. E. Gershwin, "Molecular mimicry and primary biliary cirrhosis: premises not promises," Hepatology, vol. 33, no. 4, pp. 771-775, 2001

[103] K. Hemminki, X. Li, K. Sundquist, and J. Sundquist, "Shared familial aggregation of susceptibility to autoimmune diseases," Arthritis and Rheumatism, vol. 60, no. 9, pp. 2845-2847, 2009.

[104] P. T. Donaldson, A. Baragiotta, M. A. Heneghan et al., "HLA class II alleles, genotypes, haplotypes, and amino acids in primary biliary cirrhosis: a large-scale study," Hepatology, vol. 44, no. 3, pp. 667-674, 2006.

[105] M. M. Mitchell, A. Lleo, L. Zammataro et al., "Epigenetic investigation of variably $\mathrm{X}$ chromosome inactivated genes in monozygotic female twins discordant for primary biliary cirrhosis," Epigenetics, vol. 6, no. 1, pp. 95-102, 2011.

[106] S. M. Rappaport, "Implications of the exposome for exposure science," Journal of Exposure Science and Environmental Epidemiology, vol. 21, no. 1, pp. 5-9, 2011.

[107] C. P. Wild, "Complementing the genome with an "exposome": the outstanding challenge of environmental exposure measurement in molecular epidemiology," Cancer Epidemiology Biomarkers and Prevention, vol. 14, no. 8, pp. 1847-1850, 2005.

[108] S. M. Rappaport and M. T. Smith, "Environment and disease risks,” Science, vol. 330, no. 6003, pp. 460-461, 2010.

[109] D. Smyk, E. I. Rigopoulou, H. Baum, A. K. Burroughs, D. Vergani, and D. P. Bogdanos, "Autoimmunity and environment: am I at risk?" Clinical Reviews in Allergy and Immunology. In press. 


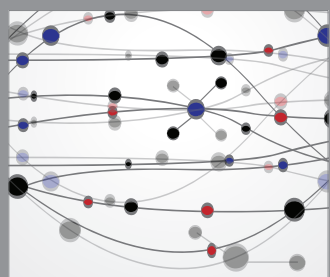

The Scientific World Journal
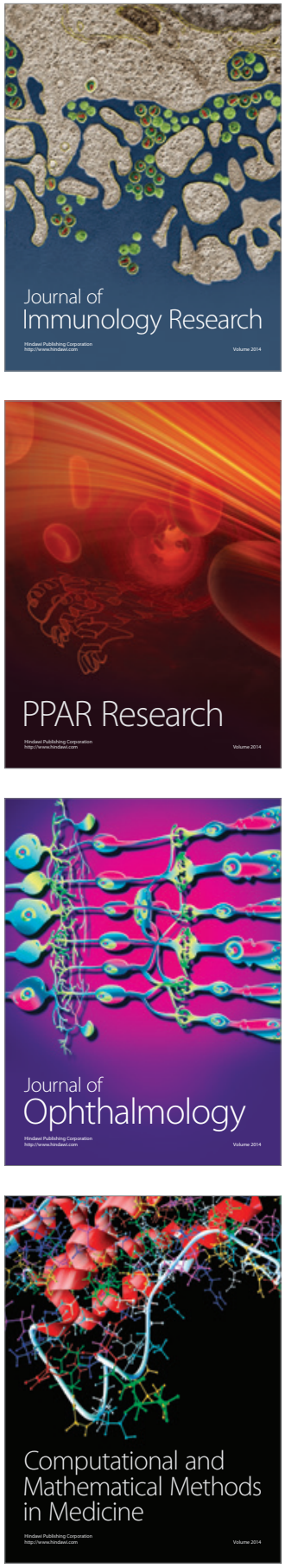

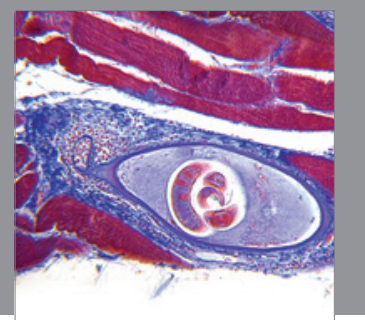

Gastroenterology

Research and Practice
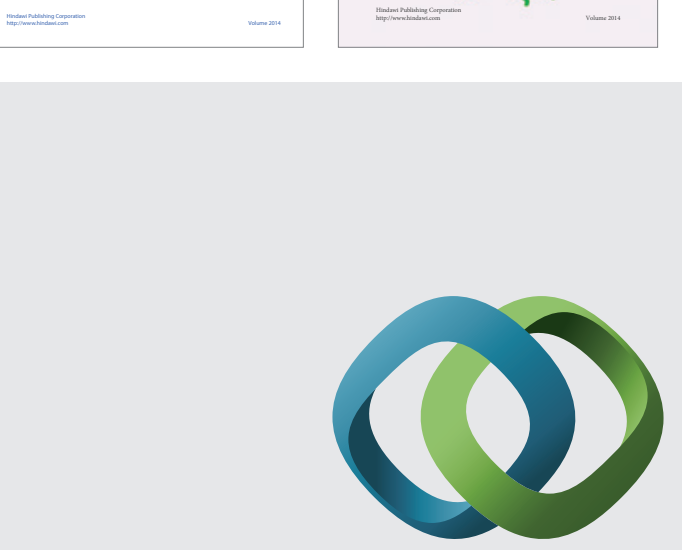

\section{Hindawi}

Submit your manuscripts at

http://www.hindawi.com
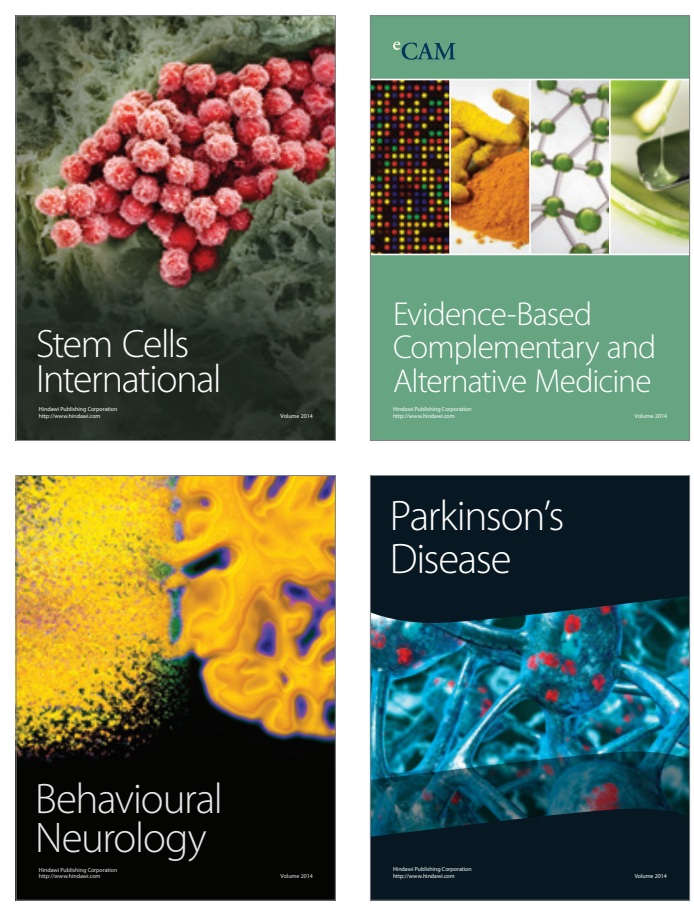

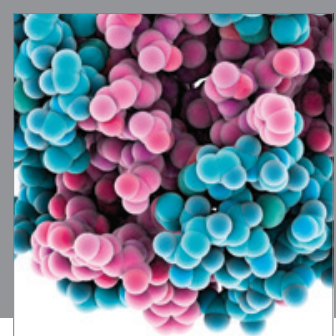

Journal of
Diabetes Research

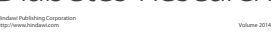

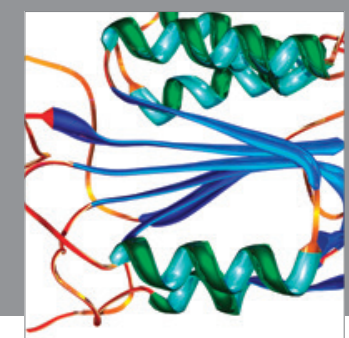

Disease Markers
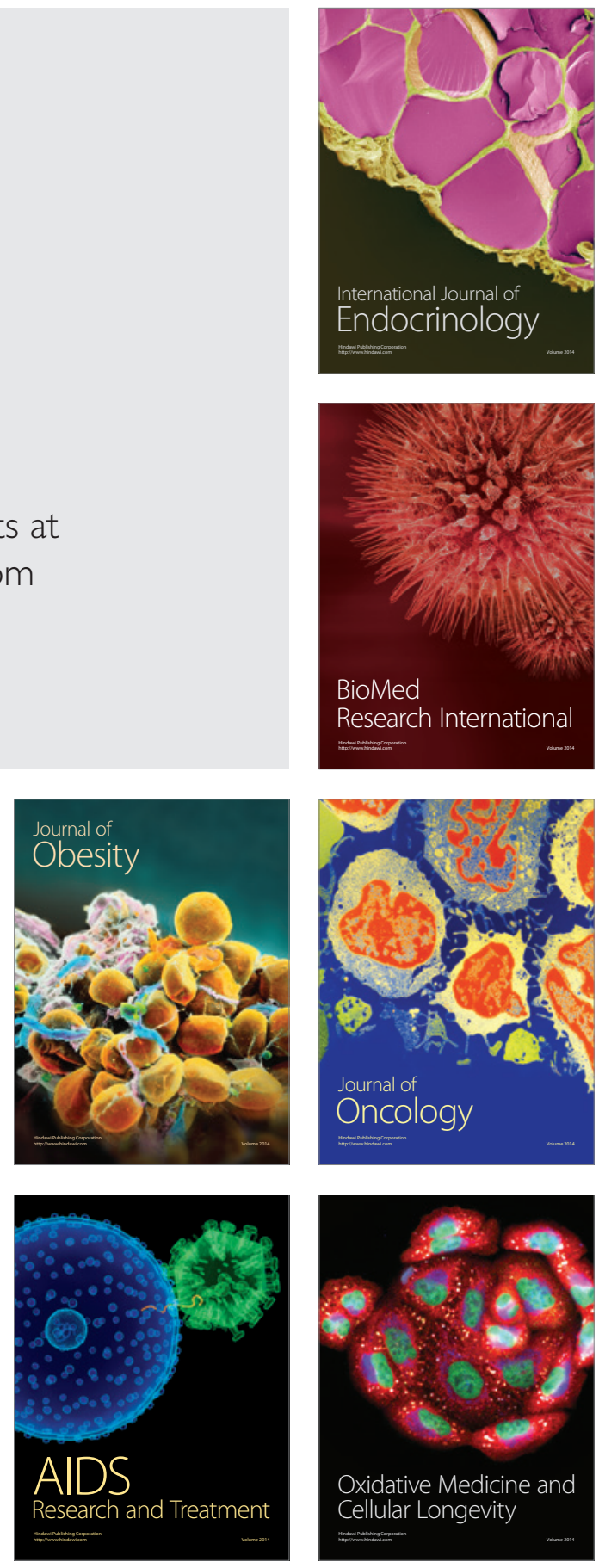